\title{
Diagnosis of a pericardial effusion with a thoracic aortic aneurysm by point-of-care ultrasound
}

\author{
Mario Francispragasam, MEd, MD*; Jeff H. Yoo, MD*; Tong V. Lam, MD*; Daniel J. Kim, $\mathrm{MD}^{*}{ }^{\dagger}$
}

\section{ABSTRACT}

We present a rare case of a young patient with chest pain whose ascending thoracic aortic aneurysm (TAA) was detected by point-of-care ultrasound (POCUS) leading to a successful surgical repair. POCUS identified a moderate pericardial effusion and an associated severely dilated ascending aorta. In this context, it is important to rule out aortic rupture and aortic dissection. We also discuss the epidemiology, complications, and management of TAAs as well as the role of cardiac POCUS in the diagnosis of thoracic aneurysmal disease.

\section{RÉSUMÉ}

Sera exposé ici un cas rare d'anévrisme de l'aorte thoracique ascendante (AATA) chez une jeune femme présentant des douleurs thoraciques, qui a été détecté par une échographie au point de service (EPS) et dont la correction chirurgicale a été couronnée de succès. L'examen a permis de visualiser un épanchement péricardique modéré en association avec une dilatation très marquée de l'aorte ascendante. Il est important, dans ce contexte, d'écarter toute possibilité de rupture de l'aorte ou de dissection de l'aorte. L'article portera sur l'épidémiologie, les complications et la prise en charge de l'AATA ainsi que sur le rôle de l'EPS cardiaque dans le diagnostic de l'anévrisme thoracique.

Keywords: thoracic aortic aneurysm, aortic aneurysm rupture, pericardial effusion, ultrasound, point-of-care ultrasound

\section{INTRODUCTION}

The rupture of a thoracic aortic aneurysm (TAA) is a rare and life-threatening event that requires early detection, risk stratification, and surgical intervention. ${ }^{1}$ Point-of-care ultrasound (POCUS) has the potential to evaluate aortic aneurysmal disease. ${ }^{2,3} \mathrm{We}$ present an unusual case where POCUS led to the early detection of a pericardial effusion with an associated ascending TAA in a 29 -year-old female.

\section{CASE REPORT}

A 29-year-old previously healthy female patient presented to the emergency department (ED) with a four-day history of pleuritic retrosternal chest pain. She had a viral upper respiratory tract infection two weeks prior to presentation. Her only medication was the oral contraceptive pill. She denied other symptoms on review of systems.

On examination, the patient appeared well, was afebrile, and had normal vital signs with a heart rate of 77 , blood pressure of $114 / 55$, respiratory rate of 16 , and room air oxygen saturation of $100 \%$. Cardiac exam revealed normal heart sounds with a grade 2 diastolic murmur at the lower sternal border. Jugular venous pressure was elevated at $6 \mathrm{~cm}$ above the sternal angle. She also had water-hammer pulses and Quincke sign. The rest of the physical examination was unremarkable.

Lab testing, including troponin, was unremarkable. Electrocardiogram revealed normal sinus rhythm, and chest X-ray showed an enlarged cardiac silhouette (Figure 1). This prompted the emergency physician (EP) to perform a bedside ultrasound, which revealed a pericardial effusion (Figure 2).

The EP consulted the emergency ultrasound fellow to scan the patient. The parasternal long axis (PSLA) window revealed a dilated ascending aorta measuring a maximum of $60 \mathrm{~mm}$ at the sinotubular aorta (Figure 3).

From the *Department of Emergency Medicine, University of British Columbia, Vancouver, BC; and tDepartment of Emergency Medicine, Vancouver General Hospital, Vancouver, BC.

Correspondence to: Dr. Daniel Kim, Vancouver General Hospital, Department of Emergency Medicine, 855 12th Avenue W., Vancouver, BC V5Z 1M9; Email: dkim000@gmail.com

(C) Canadian Association of Emergency Physicians 


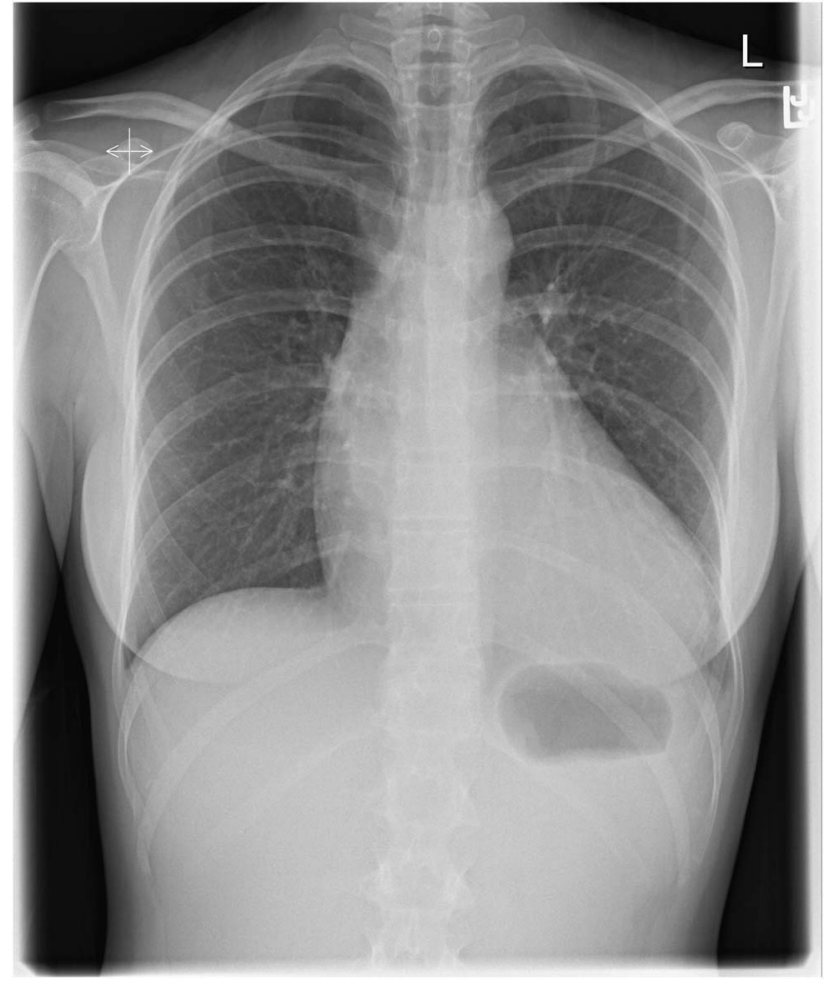

Figure 1. Chest X-ray showing an enlarged cardiac silhouette.

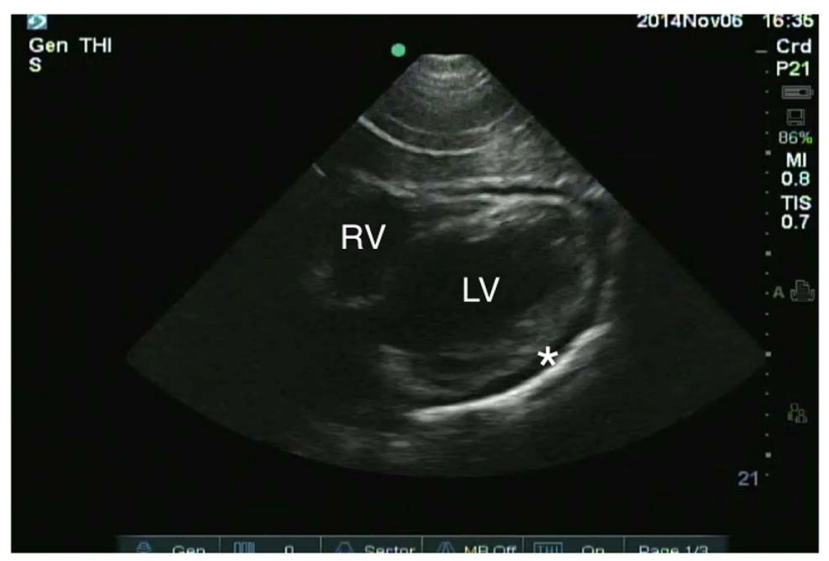

Figure 2. Subxiphoid window reveals a pericardial effusion (asterisk). The aortic root is not visible in this window. The left ventricle (LV) and right ventricle (RV) are also marked.

The previously identified circumferential pericardial effusion measured $13 \mathrm{~mm}$ at the apex. There was no evidence of tamponade physiology such as right atrial or right ventricular collapse. The inferior vena cava had preserved respiratory variation, and interrogation of the abdominal aorta revealed no further aneurysmal disease.

Consultative transthoracic echocardiography (TTE) confirmed the findings of the POCUS scan and also

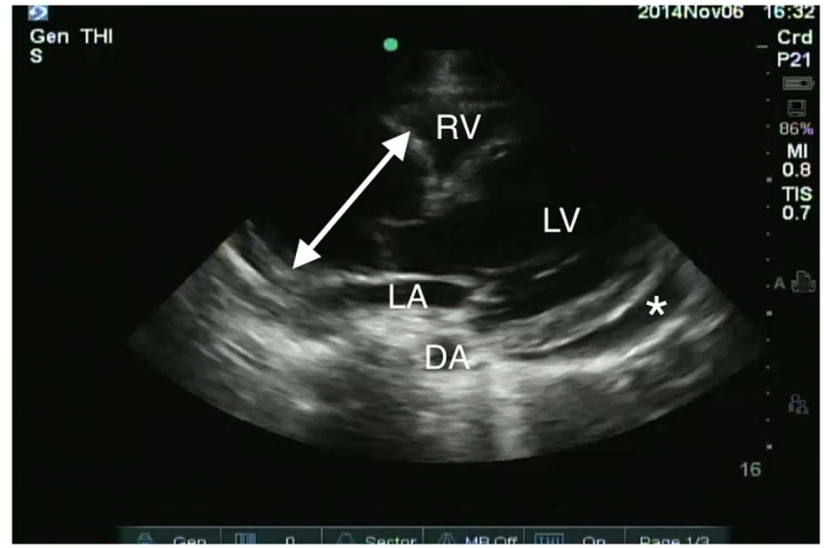

Figure 3. Parasternal long axis window demonstrates a dilated aortic root measuring $60 \mathrm{~mm}$ at the sinotubular junction (double-headed arrow) and a pericardial effusion (asterisk). The left ventricle (LV), left atrium (LA), right ventricle (RV), and descending aorta (DA) are also marked.

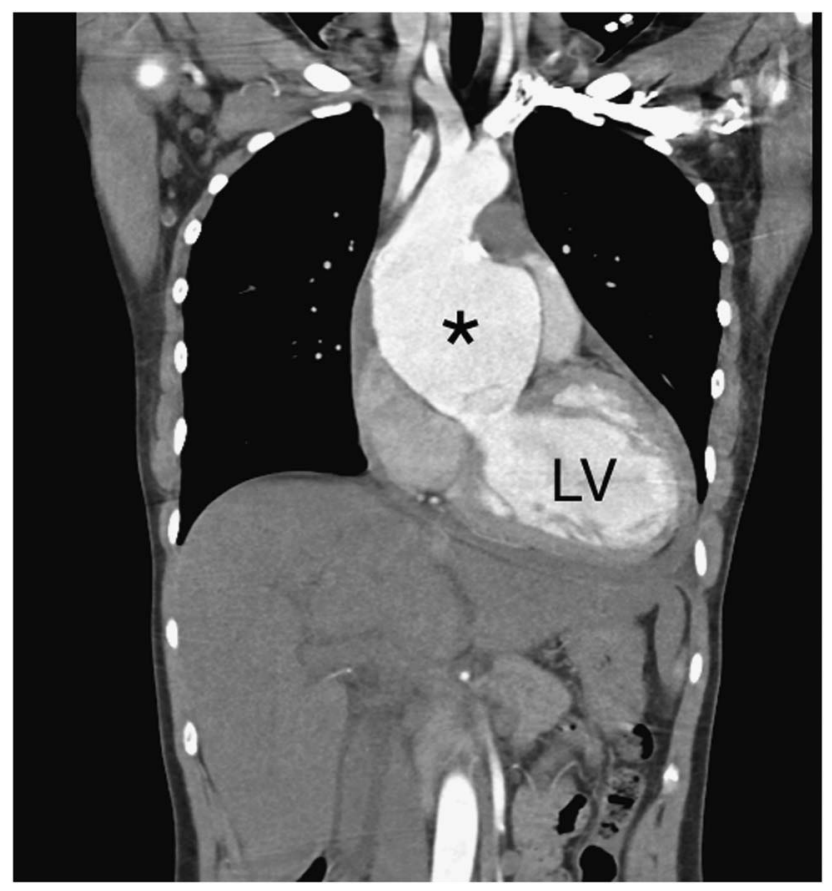

Figure 4. Coronal cut from the computed tomography angiogram demonstrates an aneurysmal ascending aorta (asterisk) with no dissection. The left ventricle (LV) is also marked.

identified severe aortic insufficiency. Computed tomography angiogram (CTA) of the chest showed a dilated aortic root measuring a maximum of $80 \mathrm{~mm}$ and a large pericardial effusion of unclear density (Figure 4). There was no aortic dissection.

The patient was given a presumptive diagnosis of pericarditis with effusion and an incidental ascending TAA. Screening bloodwork for autoimmune and 
inflammatory disease was negative. She did not meet clinical criteria for the diagnosis of any connective tissue disorders, including Marfan, Loeys-Dietz, and Ehlers-Danlos syndromes. She was admitted by the cardiovascular surgery team for further evaluation and a semi-elective repair of the aortic valve and annulus.

On the patient's third admission day, she experienced a presyncopal episode secondary to obstructive shock necessitating emergency operative repair. A cardiologyperformed TTE at that time confirmed significant tamponade physiology, so she was emergently taken to the operating room. The pericardium was opened and drained dark, old blood. The aorta was found to be very thin at the greater curvature, and a small puncture was seen in the aorta. These findings suggest the diagnosis of a contained ascending TAA rupture. She underwent successful operative repair and was ultimately discharged home in stable condition. Her native aortic valve and aorta were sent for pathologic analysis, which revealed nonspecific fibromyxoid degeneration and cystic degeneration with superimposed atherosclerosis, respectively.

\section{DISCUSSION}

An aneurysmal vessel is one that is dilated more than $150 \%$ for what is expected based on age, sex, and body weight. ${ }^{1}$ TAAs have an estimated incidence of 5.6-10.4 cases per 100,000 patient years. ${ }^{4}$ Various etiologies can cause a TAA, including degeneration associated with increased age, atherosclerosis, genetically triggered syndromes (like bicuspid aortic valve, Marfan, LoeysDietz, Ehlers-Danlos, and Turner syndromes), aortitis, trauma, and chronic aortic dissection. ${ }^{5}$ The most dangerous complications are aortic rupture and aortic dissection, which become higher risk entities when the diameter of the ascending aorta increases above 50-60 mm. ${ }^{6}$

A contained rupture of an ascending TAA is a very rare occurrence. The incidence of this event is estimated to be 5 in $100,000 .^{7}$ According to some reports, only about $41 \%$ of patients with a ruptured TAA are alive on arrival to hospital. Unfortunately, this vascular catastrophe is almost always fatal with a mortality of $97 \%-100 \%$, even in the hands of very experienced cardiovascular surgeons. ${ }^{7}$ Whereas overt, free rupture leads to massive bleeding, contained rupture is characterized by a perivascular hematoma that is sealed off by periaortic structures, such as the pleura, pericardium, and surrounding organs. As a result, patients with contained aortic rupture are hemodynamically stable. ${ }^{8}$ A finding of ascending TAA with a pericardial effusion should always raise a high clinical suspicion for aortic rupture or aortic dissection and prompt immediate surgical evaluation. ${ }^{9}$

The PSLA approach is the most useful window for diagnosing an ascending TAA using ultrasound. A phased array cardiac probe is selected because it has a small footprint that allows ultrasound waves to be directed between the ribs. The PSLA window is generated by placing the probe in the fourth or fifth intercostal space adjacent to the sternum with the probe indicator pointing towards the patient's right shoulder at 9 or 10 o'clock. Using the emergency medicine convention, the probe marker will be on the left side of the screen. If using the cardiology scanning convention, the probe marker will be on the right of the screen. The resulting image will be flipped in the horizontal plane. The operator can use either approach, provided that he or she understands the echocardiographic anatomy. This window will visualize the majority of the left ventricle, right ventricular outflow tract, left atrium, mitral and aortic valves, and aortic root. The probe can then be moved up one or two interspaces to better visualize the ascending aorta. ${ }^{10}$

Transesophageal echocardiography (TEE) is preferred over TTE for imaging the ascending aorta, but it may not be feasible, depending on adequate airway protection, need for sedation, and operator experience. TTE is often more practical in emergency situations because it is noninvasive, rapid, repeatable, and can be learned with a moderate amount of training. ${ }^{10,11}$ Aortic dilation is defined as an aortic diameter equal to or greater than $40 \mathrm{~mm}$ and aneurysm as a diameter equal to or greater than $45 \mathrm{~mm} .{ }^{8}$ These measurements should be taken in end-diastole using the leading edge technique (diameter measured from leading edge to leading edge) at the largest visible portion of the ascending aorta. ${ }^{2,3,12,13}$ POCUS demonstrates good agreement with CTA measurements of maximal thoracic aortic diameter. Using a cutoff of $40 \mathrm{~mm}$, POCUS has a sensitivity of $77 \%-79 \%$ and a specificity of $93 \%-95 \%$ for thoracic aortic dilation., ${ }^{2,3}$

Thoracic CTA is the current gold-standard imaging modality to evaluate patients who are suspected of having a TAA because it produces images that both quantify the aneurysm and allow for surgical planning., 8 Ultrasound is primarily used as a surveillance tool in 
evaluating patients with connective tissue disease who are at higher risk for TAA. ${ }^{9}$ However, in the ED, EPs can use POCUS as an initial screening tool in the assessment of patients who present with a clinical picture concerning for TAA., ${ }^{2,3}$

\section{CONCLUSION}

POCUS is a useful adjunct for evaluating high-risk patients with chest pain and can help identify lethal intrathoracic pathology, such as pericardial effusion or TAA at the bedside. CTA remains the imaging modality of choice for the diagnosis, evaluation, and surgical planning of a suspected TAA. However, in emergency situations, cardiac POCUS using a PSLA window can provide rapid recognition of an ascending TAA. This may allow for earlier diagnosis and expedited surgical management.

Competing interests: None declared.

\section{REFERENCES}

1. Elefteriades JA. Thoracic aortic aneurysm: reading the enemy's playbook. Curr Probl Cardiol 2008;33(5):203-77.

2. Taylor RA, Oliva I, Van Tonder R, et al. Point-of-care focused cardiac ultrasound for the assessment of thoracic aortic dimensions, dilation, and aneurysmal disease. Acad Emerg Med 2012;19(2):244-7.

3. Nazerian P, Vanni S, Morello F, et al. Diagnostic performance of focused cardiac ultrasound performed by emergency physicians for the assessment of ascending aorta dilation and aneurysm. Acad Emerg Med 2015;22(5):536-41.
4. Clouse WD, Hallett JW Jr, Schaff HV, et al. Improved prognosis of thoracic aortic aneurysms: a population-based study. FAMA 1998;280(22):1926-9.

5. Booher AM, Eagle KA. Diagnosis and management issues in thoracic aortic aneurysm. Am Heart 7 2011;162(1):38-46.e1.

6. Elefteriades JA, Farkas EA. Thoracic aortic aneurysm clinically pertinent controversies and uncertainties. $7 \mathrm{Am}$ Coll Cardiol 2010;55(9):841-57.

7. Johansson G, Markström U, Swedenborg J. Ruptured thoracic aortic aneurysms: a study of incidence and mortality rates. 7 Vasc Surg 1995;21(6):985-8.

8. Erbel R, Aboyans V, Boileau C, et al. 2014 ESC guidelines on the diagnosis and treatment of aortic diseases: document covering acute and chronic aortic diseases of the thoracic and abdominal aorta of the adult. Eur Heart 7 2014;35(41): $2873-926$.

9. Hiratzka LF, Bakris GL, Beckman JA, et al. 2010 ACCF/ AHA/AATS/ACR/ASA/SCA/SCAI/SIR/STS/SVM guidelines for the diagnosis and management of patients with thoracic aortic disease: a report of the American College of Cardiology Foundation/American Heart Association Task Force on Practice Guidelines, American Association for Thoracic Surgery, American College of Radiology, American Stroke Association, Society of Cardiovascular Anesthesiologists, Society for Cardiovascular Angiography and Interventions, Society of Interventional Radiology, Society of Thoracic Surgeons, and Society for Vascular Medicine. Circulation 2010;121(13):e266-369.

10. Peterson D, Arntfield RT. Critical care ultrasonography. Emerg Med Clin North Am 2014;32(4):907-26.

11. Evangelista A, Flachskampf FA, Erbel R, et al. Echocardiography in aortic diseases: EAE recommendations for clinical practice. Eur 7 Echocardiogr 2010;11:645-58.

12. Vriz O, Driussi C, Bettio M, et al. Aortic root dimensions and stiffness in healthy subjects. Am $\mathcal{F}$ Cardiol 2013;112(8): 1224-9.

13. Roman MJ, Devereux RB, Kramer-Fox R, et al. Twodimensional echocardiographic aortic root dimensions in normal children and adults. Am 7 Cardiol 1989;64(8):507-12. 